\title{
Principles Derived from Neurolinguistics of Brain for Design of Translation Machines
}

\author{
Zi-Jian Cai \\ CaiFortune Company, Suzhou, China \\ Email: hrsh8@126.com
}

Received 2 May 2016; accepted 5 June 2016; published 8 June 2016

Copyright (C) 2016 by author and OALib.

This work is licensed under the Creative Commons Attribution International License (CC BY). http://creativecommons.org/licenses/by/4.0/

(c) (i) Open Access

\section{Abstract}

Recently, it was extended by the author the declarative/procedural model to a new semantic/ syntactic/episodic model for language, extended to encompass the sentential meanings to the neural linguistic processes. In this article, it is applied this new semantic/syntactic/episodic model of brain to derive three feasible principles to direct machine translation respectively. First, it is necessary to establish the dictionary for translation of words and phrases. Second, it is also necessary to read out the grammar of language to be translated from and to comply with the grammar of language to be translated into, arranging such parts of speech as noun, verb and adjective into order. Third, it is in further necessary to determine one correct meaning of some words of multiple meanings by matching them with statistical associations with others. Whereas, due to the lack of scientific guidance from neurolinguistics, it has mostly been adopted two linguistic processes in the present machine translation, either with only word and grammar translation, or with only word and statistical translation, and therefore has been unsatisfactory. Through comparison, it is pointed out that the machine translation with three principles would exceed the human brain in all three linguistic aspects respectively. In this regard, herein it is newly formulated the three principles derived from the semantic/syntactic/episodic neurolinguistic model of brain for machine translation. Prospectively, it is a significant progression leading the new technological leap of artificial intelligence with acquisition of ability in natural language equal to and even superior to that of human brain.

\section{Keywords}

Language, Three Neurolinguistic Processes, Machine Translation Principle, Semantic Dictionary, Grammar, Statistical Translation

Subject Areas: Artificial Intelligence, Linguistics, Neuroscience 


\section{Introduction}

Up to now, native language is the high and complex mental activity unique to humans for communication and meditation. Recently, the author extended the present declarative/procedural model underlying two kinds of neural processes to a new semantic/syntactic/episodic model of three kinds of neural processes able to encompass the sentential meanings to the neural linguistic processes [1].

One of the most important applications for illustration of neural processes in brain underlying language lies in the promotion of artificial intelligence to assimilate or improve linguistic processes as that in human brain. At present, machine translation is the most relevant artificial linguistic processing automation prospectively utilized widely in various situations. Whereas, the results of present machine translation have been quite unsatisfactory, and translational mistakes occur everywhere [2]-[5], less competent to that of human persons with professional training in translation. Due to the lack of scientific guidance from neurolinguistics, machine translation has mostly adopted two linguistic processes up to now, either with only word and grammar translation, or with only word and statistical translation.

With the recent extension of declarative/procedural model of two neurolinguistic processes to the semantic/ syntactic/episodic model of three neurolinguistic processes [1], it is possible to improve the machine translation with the three neurolinguistic processes of brain instead of two. In this article, it is attempted to in further consider this application, and to compare the consequential machine translation to that of human brain.

\section{Theoretical Background: The Semantic/Syntactic/Episodic Three Neurolinguistic Processes of Brain}

\subsection{Word Comprehension as Memory Associations in Brain}

Pinker, Ullman and so on integrated a declarative/procedural model for linguistic processing in brain. They suggested that the linguistic lexicon of word-specific knowledge should subserve storage of meanings of words and phrases, and depend on the temporal-lobe substrates of declarative memory underlying the storage and usage of facts and events [1] [6]-[8].

It is known that memory can be divided into declarative memory for facts or episodes, and procedural memory for skills or habits. Declarative memory can be divided into short-term memory and long-term memory. Shortterm memory is the memory of precedent episodes or facts within minutes or seconds, while long-term memory is the memory of them more than hours or days ago. Besides, remote (retrograde) memory is the long term memory lasting for decades of years. This classification of memories has been illustrated early in numerous reviews [9] and books [10].

Words in native language are acquired as declarative memories during childhood, so that they are utilized as stable remote retrograde memories in adults [1]. All words have one or several defined specific meanings, so that the words in native language are actually the remote memories with association with one or several specific meanings [1] [6]-[8]. The words are actually the associative memories as one sort of remote declarative memories stored in the brain.

Some derivatives of words are formed from linguistic rules instead of learned from teaching [6] [7]. Nonetheless, word derivatives are also associated with original words or specific meanings, so that they are also present as memory associations in cortices [1].

There are many neuropsychological evidences in support of this suggestion in many reviews [1] [6]-[8]. More typical in consistency in addition, those patients with variations in locations of stroke manifest many specific subtypes of aphasias, including anomic aphasia and transcortical sensory aphasia [11]. Besides, alexia is characterized as dysfunction in word comprehension during reading with lesion in fusiform gyrus or nearby cortical regions [12]-[14], whereas anomic aphasia is characterized as dysfunction in appropriate naming with lesion in anterior temporal lobe [15]-[17]. Obviously, word comprehension is stored as remote memory association of specific cortical modality in brain.

\subsection{Grammar as Procedural Memory in Brain}

In the declarative/procedural neurolinguistic model postulated by Pinker, Ullman and so on, it was suggested that the linguistic grammar should subserve the sequential combination of lexical items by procedural rules into complex sentences, and depend on a network of specific frontal, parietal, basal-ganglia and cerebellar structures 
of procedural memory in the brain [1] [6]-[8]. In syntax, the linguistic grammar arranges such parts of speech as noun, verb and adjective into order to form the sentence.

There are also many neuropsychological evidences in support of this suggestion in many reviews [1] [6]-[8]. More typical in consistency in addition, there are present a subtype of patients called non-fluent/agrammatic aphasia specially characterized as poor in grammatical comprehension and expression [18]. In pathology, nonfluent/agrammatic aphasia is associated with frontotemporal degeneration from tauopathy [18], different from semantic aphasia with frontotemporal degeneration from TDP-43 proteinopathy, and semantic aphasia with Alzheimer pathology [18] [19]. Besides, it was demonstrated that agrammatic aphasia could be distinguished from semantic aphasia with detailed diagnosis [20] [21], such as Parkinsonian motor features [20]. Further, it was more importantly reported that some agrammatic aphasic patients were also impaired on artificial grammar learning as compared to controls [22], indicating the breakdown of language in agrammatic aphasia to be associated with damage to those neural structures concurrently subserving both native language and artificial procedural learning of sequence.

\subsection{Episodic Cortical Coordination in Brain}

Recently, the author extended the declarative/procedural model underlying two neurolinguistic processes to a new semantic/syntactic/episodic model of three neurolinguistic processes in brain [1]. It was added that, besides word comprehension as remote declarative associative memory while grammatical rule as procedural memory in brain, the episodic modality coordination of cortices was also required for language performance and organization in addition [1]. Extension of declarative/procedural model to semantic/syntactic/episodic model helped encompass the sentential meanings to the neural linguistic processes [1].

There are similarly many neuropsychological evidences in support of this suggestion in many articles and reviews [1], as in the followings: 1) The dopaminergic system may be involved in the linguistic modality disorganization, as the dopaminergic antagonists [23] [24] alleviate while dopaminergic genes [25] phenotype stuttering. 2) From drugs in many assisting therapies of aphasia, it is implicated that the ascending cholinergic system, noradrenergic projections and nonspecific activating projections from intralaminar thalamic nuclei may participate in episodic coordination of cortical modalities in linguistic processes [26] [27]. 3) It is evidenced that the gamma bands of high frequency, subject to modulation by the ascending cholinergic systems, associate with the word congruency at sentential level [28]-[30] as well as the episodic coordination of cortical modalities. 4) The characteristics in ontogenetic development of bilinguals indicate that there must be difference in episodic coordination of cortical modalities for the two types of bilinguals acquired before and after 6 years old at age [31].

\subsection{The Semantic/Syntactic/Episodic Neurolinguistic Brain Processes}

In all, following the processes of language from simple word to complex sentence, herein it is outlined the semantic/syntactic/episodic model to organize the linguistic processes in brain [1]. Word comprehension in adult is characterized as remote retrograde memory in native language, with stable association between the word and its meanings, so that manifests as declarative memory associations. Whereas, the grammar actually represents a set of linguistic procedures, in which the words are sequentially arranged to form the sentence according to their characteristics as noun, verb, adjective and so on. Furthermore, the modality coordination of words episodic in meanings in sentences is accomplished by the ascending dopaminergic, cholinergic and noradrenergic systems. In these respects, semantic memory association, procedural grammar syntax and episodic modality coordination interact to organize the language from simple word to complex sentence. This is exactly the semantic/syntactic/ episodic model with three kinds of neurolinguistic processes in brain.

\section{Principles Derived from Brain Neurolinguistics for Machine Translation}

\subsection{The Present Situations of Machine Translation}

At present, there are two types of machine translation, adopting two different strategies of design respectively. One is early in method following which to translate individual words and grammar of sentence [2] [3]. The difficulty of this type of machine translation lies in that many words have multiple meanings, so that the translation often makes mistakes due to this reason. Another is the recent method called as statistical machine translation [4] 
[5], in which machine translation is made according to the statistical concurrence of words and contents. The difficulty of this type of machine translation lies in that the statistical concurrence of words and contents is limited from collection, while the real objects of translation are limitless, so that the machine translation is not able to cover all fields of translation. Both types of machine translation have only adopted two linguistic processes in brain, either with only word and grammar translation, or with only word and statistical translation.

Because of the lack of scientific guidance from neurolinguistics, the present machine translation has mostly chosen one of the two types of methods, and therefore only adopted two linguistic processes rather than the recently revealed three brain linguistic processes. In this regard, the results of machine translation are quite unsatisfactory at present, and translational mistakes occur everywhere [2]-[5].

\subsection{Two Mathematical Rules Utilized in Machine Translation}

There are also mainly two mathematical principles utilized in present machine translation. One is Markov Process, while another is Bayesian Rule.

The Markov Process describes a kind of status in series, among which the probability of one status being determined by finite numbers of previous ones. The Markov Process is expressed as the following mathematical equation:

$$
P\left(X_{n+1}=x \mid X_{1}=x_{1}, X_{2}=x_{2}, \cdots, X_{n}=x_{n}\right)=P\left(X_{n+1}=x \mid X_{n}=x_{n}\right) .
$$

Because the language contains huge numbers of words and phrases, as well as many words of multiple meanings, the Markov Process does not fit most situations of machine translation. Whereas, the Markov Process has been typically adopted by some authors for finite voice recognition [32] and feature recognition [33] in language processing.

On the other hand, the Bayesian Rule describes the method to judge the probability of an event according to its previous probability of occurrence, in which the present probability can even be in further modulated by the addition of occurrence of present events. The Bayesian Rule is expressed as the following mathematical equation:

$$
P(h \mid d)=\frac{P(d \mid h) P(h)}{\sum_{i} P\left(d \mid h_{i}\right) P\left(h_{i}\right)}
$$

Since Bayesian Rule can determine the probability of the meaning of a word of multiple meanings with its previous probability of concurrence with others, while can even adjust the probability of words in reservoir with their later occurrence, Bayesian Rule is very suitable for statistical translation, and widely utilized in present machine translation [2].

\subsection{Three Principles Derived from Brain Neurolinguistics for Machine Translation}

With the recent illustration of the semantic/syntactic/episodic model of three neurolinguistic processes [1], it is possible to derive three corresponding principles from them for guidance of software design of translation machines.

From word comprehension as remote declarative memory associations in brain, it can be formulated the first principle for design of translation machines as followings:

Principle 1: Corresponding to the word comprehension as memory associations, it is necessary to establish the dictionary for translation of words and phrases in translation machine.

From grammatical rule as procedural memory in brain, it can be formulated the second principle for design of translation machines as followings:

Principle 2: Corresponding to the grammatical rule as procedural memory, it is necessary to install the linguistic grammar for reading and forming sentence from words and phrases in translation machine, arranging such parts of speech as noun, verb and adjective into order.

From episodic coordination of language in brain, it can be formulated the third principle for design of translation machines as followings:

Principle 3: Corresponding to the episodic coordination of language, it is necessary to check with statistics of concurrence for the words and phrases of multiple meanings, and figure out one correct meaning of them in translation machine. 
The three principles from brain neurolinguistics for guidance of software design of translation machines can be outline in Table 1.

\section{Consequential Improvements}

To understand the consequences brought about by the three principles of machine translation derived from brain neurolinguistics, it is necessary to compare the translation of machine to that of human brain.

As the translation machines are improved gradually in the world, while different companies may adopt different software tools, it is not statistically meaningful to compare the translation of any individual machine to that of human brain. Whereas, through comparison with that of human brain in the ability of translation, it is possible to figure out the consequential improvements of the feasible machine translation following the three principles derived from neurolinguistics.

For Principle 1 corresponding to the word comprehension as memory associations to establish the dictionary for translation of words and phrases, the quantity and quality in meanings of words and phrases can be both higher in machine dictionary than that stored in any human brain. In this respect, it is definite that the machine translation would be superior than that of human brain on this aspect.

For Principle 2 corresponding to the grammatical rule as procedural memory to install the linguistic grammar in machine, the quality of grammar checking have not matched to that of humans nowadays in some softwares. Whereas, with the limited contents of linguistic grammar and with the improvement of software, it is definite that the translation machine would certainly match and exceed in ability of grammar than that of human brain in future.

For Principle 3 corresponding to the episodic coordination of language to statistically check and figure out the correct meanings of words and phrases of multiple meanings, the translation machine and human brain accumulate the statistics of word concurrence in different ways. In translation machine, statistics of various word associations is accumulated through modification of previous probability with encountered new occurrence of associations following Bayesian Rule. Whereas in human brain, statistics of various word associations is accumulated through life-long learning and experiencing. It is necessary to point out that, if the translation machine is run at its leisure time by the owner for professional statistical accumulation of various word associations, while the human brain is oriented to irrelevant livelihoods in most time, the translation machine would exceed that of human brain in statistical accumulation of various word associations for professional translation.

It is plausible to doubt that, although the machine translation is able to exceed that of human brain in all of the three aspects following the three derived principles, it is still possible that the human brain may resort to other structures for language processing and translation, so that may be still more superior than translation machine during translation.

This doubt can be solved by inspection of all relevant brain structures to language processing. In the semantic/ syntactic/episodic model of neurolinguistics, the following brain structures are adopted: 1) Word comprehension is processed in the temporal cortices for remote declarative memory storage [1] [6]-[8]. 2) Grammatical rule is processed in the frontal, parietal, basal-ganglia and cerebellar structures of procedural memory in brain [1] [6]-[8]. 3) Episodic coordination of linguistic cortices is accomplished by the ascending reticular dopaminergic, cholinergic and noradrenergic systems [1].

Beyond these structures, in brain there are only the limbic structures responsible for processing of emotions [9]-[36], and right hemisphere responsible for processing of information other than language in healthy subjects [31] [37], such as spatial and emotional information. Such nonlinguistic information is usually processed along with language as association with semantic or sentential contents in meanings, and is dealt with and covered well by Principle 3 for machine translation. In this regard, there is no doubt that, if professionally trained following Principle 3, the translation machine with the three principles derived from brain neurolinguistics would exceed the human brain in ability of language translation.

Table 1. Three principles from brain neurolinguistics for software design of machine translation.

\begin{tabular}{ccc}
\hline & Correspondents in brain & Guiding principles for software \\
\hline Principle 1 & Words as memory associations & Make dictionary of words/phrases \\
Principle 2 & Grammar as procedural memory & Install linguistic grammar \\
Principle 3 & Episodic coordination of language & Check word meaning statistically \\
\hline
\end{tabular}




\section{Feedback from Other Parallel Progressions}

It is noticed that improvement of machine translation is just one of the major achievements for the semantic/ syntactic/episodic neurolinguistic model of brain to bring about. There are many things in other fields which can be significantly improved in parallel, as followings: 1) Improvement of mutual interaction and comprehension between human and machine. 2) Improvement of language ability of artificial robot. 3) Improvement of language ability of characters or actors/actresses in computer games. 4) Improvement of detection, interpretation and comprehension of brain activities [38]. 5) Improvement of psychoanalysis and psychological consultation [36].

The parallel progressions in other relevant fields would certainly feedback to improve the technology of machine translation. Especially, the perspective achievements in comprehension of language in various voices and situations by the machine can directly feedback to modulate the statistical probability in Principle 3 . With the parallel progressions in many fields, it would be predicted that the ability of machine translation would advance faster than herein expected.

\section{Perspectives}

With regard to the consequential improvements that, if well trained professionally, the translation machine can exceed the human brain in ability of language translation, as well as the parallel progressions that would also be achieved in many relevant fields else, it is obvious that the new technological era with automation of language processing and translation would come to the life and work of everybody and everyday. This is the great contribution from the neurolinguistics of brain to the progression of modern civilization.

\section{Conclusions}

In conclusion, in this article, from the three neurolinguistic semantic/syntactic/episodic model of brain, it is derived three principles for guidance of software design of translation machines, as followings: 1) Principle 1 is the necessity to establish the dictionary for translation of words and phrases in translation machine. 2) Principle 2 is the necessity to install the linguistic grammar for reading and forming sentence from words and phrases in translation machine, arranging such parts of speech as noun, verb and adjective into order. 3) Principle 3 is the necessity to check with statistics for the words and phrases of multiple meanings and figure out one correct meaning of them in translation machine.

In this article, it is also demonstrated the consequential improvements of feasible translation machine following the three principles from neurolinguistics by comparing their ability of translation with that of human brain. For Principle 1 to establish the dictionary for translation of words and phrases, the quantity and quality in meanings of words and phrases can be both higher in machine dictionary than that stored in human brain. For Principle 2 to install the linguistic grammar in machine, with the limited contents of linguistic grammar and with the improvement of software, it is definite that the translation machine would certainly match and exceed in ability of grammar than that of human brain. For Principle 3 to statistically check and figure out the correct meaning of words and phrases of multiple meanings, if the translation machine is trained at its leisure time by the owner for professional statistical accumulation of various word associations following Bayesian Rule, while the human brain is oriented to irrelevant livelihoods in most time, the translation machine would exceed that of human brain in statistical accumulation of various word associations for professional translation.

In perspective, it is obvious that, with the contribution from the neurolinguistic progressions of brain, the new technological era with automation of language processing and translation would come to the life and work of everybody and everyday.

\section{Conflict of Interest}

The author declares neither conflict of interest nor financial support for this work.

\section{References}

[1] Cai, Z.-J. (2015) Semantic Memory Association, Procedural Grammar Syntax and Episodic Modality Coordination as Three Interactive Neural Processes Organizing Language: A Model. Open Access Library Journal, 2, e1718. http://dx.doi.org/10.4236/oalib.1101718 
[2] Zhang, M., Duan, X.Y. and Chen, W.L. (2014) Bayesian Constituent Context Model for Grammar Induction. IEEE/ ACM Transactions on Audio, Speech, and Language Processing, 22, 531-541. http://dx.doi.org/10.1109/TASLP.2013.2294584

[3] Zhang, M., Che, W.X., Zhou, G.D., Aw, A., Tan, C.L., Liu, T. and Li, S. (2008) Semantic Role Labeling Using a Grammar-Driven Convolution Tree Kernel. IEEE Transactions on Audio, Speech, and Language Processing, 16, 13151329. http://dx.doi.org/10.1109/TASL.2008.2001104

[4] Xiong, D.Y., Zhang, M. and Li, H.Z. (2011) A Maximum-Entropy Segmentation Model for Statistical Machine Translation. IEEE Transactions on Audio, Speech, and Language Processing, 19, 2494-2505. http://dx.doi.org/10.1109/TASL.2011.2144971

[5] Xiong, D.Y., Zhang, M. and Wang, X. (2015) Topic-Based Coherence Modeling for Statistical Machine Translation. IEEE/ACM Transactions on Audio, Speech, and Language Processing, 23, 483-493. http://dx.doi.org/10.1109/TASLP.2015.2395254

[6] Pinker, S. (1991) Rules of language. Science, 253, 530-535. http://dx.doi.org/10.1126/science.1857983

[7] Ullman, M.T., Corkin, S., Coppola, M., Hickok, G., Growdon, J.H., Koroshetz, W.J. and Pinker, S. (1997) A Neural Dissociation within Language: Evidence That the Mental Dictionary Is Part of Declarative Memory, and That Grammatical Rules Are Processed by the Procedural System. Journal of Cognitive Neuroscience, 9, 266-276. http://dx.doi.org/10.1162/jocn.1997.9.2.266

[8] Ullman, M.T. (2004) Contributions of Memory Circuits to Language: The Declarative/Procedural Model. Cognition, 92, 231-270. http://dx.doi.org/10.1016/j.cognition.2003.10.008

[9] Cai, Z.-J. (1990) The Neural Mechanism of Declarative Memory Consolidation and Retrieval: A Hypothesis. Neuroscience \& Biobehavioral Reviews, 14, 295-304. http://dx.doi.org/10.1016/S0149-7634(05)80039-9

[10] Squire, L.R. (1987) Memory and Brain. Oxford University Press, Oxford.

[11] Yang, Z.H., Zhao, X.Q., Wang, C.X., Chen, H.Y. and Zhang, Y.M. (2008) Neuroanatomic Correlation of the PostStroke Aphasias Studied with Imaging. Neurological Research, 30, 356-360. http://dx.doi.org/10.1179/174313208X300332

[12] Turkeltaub, P.E., Goldberg, E.M., Postman-Caucheteux, W.A., Palovcak, M., Quinn, C., Cantor, C. and Coslett, H.B. (2014) Alexia Due to Ischemic Stroke of the Visual Word Form Area. Neurocase, 20, 230-235. http://dx.doi.org/10.1080/13554794.2013.770873

[13] Leśniak, M., Soluch, P., Steppień, U., Czepiel, W. and Seniów, J. (2014) Pure Alexia after Damage to the Right Fusiform Gyrus in a Right-Handed Male. Neurologia I Neurochirurgia Polska, 48, 373-377. http://dx.doi.org/10.1016/j.pjnns.2014.09.003

[14] Kleinschmidt, A. and Cohen, L. (2006) The Neural Bases of Prosopagnosia and Pure Alexia: Recent Insights from Functional Neuroimaging. Current Opinion in Neurology, 19, 386-391. http://dx.doi.org/10.1097/01.wco.0000236619.89710.ee

[15] Hanley, J.R. (2014) Accessing Stored Knowledge of Familiar People from Faces, Names and Voices: A Review. Frontiers in Bioscience (Elite Edition), 6, 198-207. http://dx.doi.org/10.2741/E702

[16] Ives-Deliperi, V.L. and Butler, J.T. (2012) Naming Outcomes of Anterior Temporal Lobectomy in Epilepsy Patients: A Systematic Review of the Literature. Epilepsy \& Behavior, 24, 194-198. http://dx.doi.org/10.1016/j.yebeh.2012.04.115

[17] Semenza, C. (2011) Naming with Proper Names: The Left Temporal Pole Theory. Behavioural Neurology, 24, $277-$ 284. http://dx.doi.org/10.1155/2011/650103

[18] Grossman, M. (2012) The Non-Fluent/Agrammatic Variant of Primary Progressive Aphasia. Lancet Neurology, 11, 545-555. http://dx.doi.org/10.1016/S1474-4422(12)70099-6

[19] Mesulam, M.M. (2013) Primary Progressive Aphasia and the Language Network: The 2013 H. Houston Merritt Lecture. Neurology, 81, 456-462. http://dx.doi.org/10.1212/WNL.0b013e31829d87df

[20] Graff-Radford, J., Duffy, J.R., Strand, E.A. and Josephs, K.A. (2012) Parkinsonian Motor Features Distinguish the Agrammatic from Logopenic Variant of Primary Progressive Aphasia. Parkinsonism \& Related Disorders, 18, 890-892. http://dx.doi.org/10.1016/j.parkreldis.2012.04.011

[21] Thompson, C.K., Cho, S., Hsu, C.J., Wieneke, C., Rademaker, A., Weitner, B.B., Mesulam, M.M. and Weintraub, S. (2012) Dissociations between Fluency and Agrammatism in Primary Progressive Aphasia. Aphasiology, 26, 20-43. http://dx.doi.org/10.1080/02687038.2011.584691

[22] Christiansen, M.H., Louise Kelly, M., Shillcock, R.C. and Greenfield, K. (2010) Impaired Artificial Grammar Learning in Agrammatism. Cognition, 116, 382-393. http://dx.doi.org/10.1016/j.cognition.2010.05.015

[23] Maguire, G.A., Yu, B.P., Franklin, D.L. and Riley, G.D. (2004) Alleviating Stuttering with Pharmacological Interven- 
tions. Expert Opinion on Pharmacotherapy, 5, 1565-1571. http://dx.doi.org/10.1517/14656566.5.7.1565

[24] Stager, S.V., Calis, K., Grothe, D., Bloch, M., Berensen, N.M., Smith, P.J. and Braun, A. (2005) Treatment with Medications Affecting Dopaminergic and Serotonergic Mechanisms: Effects on Fluency and Anxiety in Persons Who Stutter. Journal of Fluency Disorders, 30, 319-335. http://dx.doi.org/10.1016/j.jfludis.2005.09.004

[25] Lan, J., Song, M., Pan, C., Zhuang, G., Wang, Y., Ma, W., Chu, Q., Lai, Q., Xu, F., Li, Y., Liu, L. and Wang, W. (2009) Association between Dopaminergic Genes (SLC6A3 and DRD2) and Stuttering among Han Chinese. The American Journal of Human Genetics, 54, 457-460. http://dx.doi.org/10.1038/jhg.2009.60

[26] Small, S.L. and Llano, D.A. (2009) Biological Approaches to Aphasia Treatment. Current Neurology and Neuroscience Reports, 9, 443-450. http://dx.doi.org/10.1007/s11910-009-0066-X

[27] Berthier, M.L., Pulvermüller, F., Dávila, G., Casares, N.G. and Gutiérrez, A. (2011) Drug Therapy of Post-Stroke Aphasia: A Review of Current Evidence. Neuropsychology Review, 21, 302-317. http://dx.doi.org/10.1007/s11065-011-9177-7

[28] Wang, L., Zhu, Z. and Bastiaansen, M. (2012) Integration or Predictability? A Further Specification of the Functional Role of Gamma Oscillations in Language Comprehension. Frontiers in Psychology, 3, 1-12. http://dx.doi.org/10.3389/fpsyg.2012.00187

[29] Vidal, J.R., Freyermuth, S., Jerbi, K., Hamamé, C.M., Ossandon, T., Bertrand, O., Minotti, L., Kahane, P., Berthoz, A. and Lachaux, J.P. (2012) Long-Distance Amplitude Correlations in the High $\gamma$ Band Reveal Segregation and Integration within the Reading Network. The Journal of Neuroscience, 32, 6421-6434. http://dx.doi.org/10.1523/JNEUROSCI.4363-11.2012

[30] Weiss, S. and Müller, H.M. (2013) The Non-Stop Road from Concrete to Abstract: High Concreteness Causes the Activation of Long-Range Networks. Frontiers in Human Neuroscience, 7, 1-13. http://dx.doi.org/10.3389/fnhum.2013.00526

[31] Hull, R. and Vaid, J. (2007) Bilingual Language Lateralization: A Meta-Analytic Tale of Two Hemispheres. Neuropsychologia, 45, 1987-2008. http://dx.doi.org/10.1016/j.neuropsychologia.2007.03.002

[32] Duarte, T., Prikladnicki, R., Calefato, F. and Lanubile, F. (2014) Speech Recognition for Voice-Based Machine Translation. IEEE Software, 31, 26-31. http://dx.doi.org/10.1109/MS.2014.14

[33] Zhou, G.D. and Jian, S. (2003) Integrating Various Features in Hidden Markov Model Using Constraint Relaxation Algorithm for Recognition of Named Entities without Gazetteers. International Conference on Natural Language Processing and Knowledge Engineering, Beijing, 26-29 October 2003, 465-470. http://dx.doi.org/10.1109/NLPKE.2003.1275951

[34] Cai, Z.-J. (1991) The Functions of Sleep: Further Analysis. Physiology \& Behavior, 50, 53-60. http://dx.doi.org/10.1016/0031-9384(91)90497-C

[35] Cai, Z.-J. (1995) An Integrative Analysis to Sleep Functions. Behavioural Brain Research, 69, 187-194. http://dx.doi.org/10.1016/0166-4328(95)00005-E

[36] Cai, Z.-J. (2015) Extending Psychoanalysis with Theories on Sleep Functions. Journal of Sleep Disorders \& Therapy, 4, Article ID: 1000217.

[37] Preibisch, C., Neumann, K., Raab, P., Euler, H.A., von Gudenberg, A.W., Lanfermann, H. and Giraud, A.L. (2003) Evidence for Compensation for Stuttering by the Right Frontal Operculum. Neuroimage, 20, 1356-1364. http://dx.doi.org/10.1016/S1053-8119(03)00376-8

[38] Cai, Z.-J. (2015) Advocacy for Extension of Microwave and Infrared to Detect the Brain Activities. Journal of Medical Diagnostic Methods, 4, Article ID: 1000188. 